\title{
Candidates for giant lobes projecting from the LBV stars P Cygni and R 143
}

\author{
J. Meaburn ${ }^{1}$, P. Boumis ${ }^{2}$, M. P. Redman ${ }^{3}$, J. A. López ${ }^{4}$, and F. Mavromatakis ${ }^{5}$ \\ 1 Jodrell Bank Observatory, University of Manchester, Macclesfield SK11 9DL, UK \\ 2 Institute of Astronomy \& Astrophysics, National Observatory of Athens, I. Metaxa \& V. Paulou, P. Penteli, GR-15236 Athens, \\ Greece \\ ${ }^{3}$ Dublin Institute for Advanced Studies, School of Cosmic Physics, 5 Merrion Square, Dublin 2, Republic of Ireland \\ 4 Instituto de Astronomia, UNAM, Apdo. Postal 877. Ensenada, BC 22800, México \\ 5 University of Crete, Physics Department, PO Box 2208, 71003 Heraklion, Crete, Greece
}

Received 19 January 2004 / Accepted 6 April 2004

\begin{abstract}
Deep, wide-field, continuum-subtracted, images in the light of the H $\alpha+[\mathrm{N}$ II] 6548 \& $6584 \AA$ and [O III] $] 5007 \AA$ nebular emission lines have been obtained of the environment of the Luminous Blue Variable (LBV) star P Cygni. A previously discovered, receding, nebulous filament along PA $50^{\circ}$ has now been shown to extend up to $12^{\prime}$ from this star. Furthermore, in the light of [O III] $5007 \AA$, a southern counterpart is discovered as well as irregular filaments on the opposite side of P Cygni. Line profiles from this nebulous complex indicate that this extended nebulosity is similar to that associated with middleaged supernova remnants. However, there are several indications that it has originated in P Cygni and is not just a chance superposition along the same sight-line. This possibility is explored here and comparison is made with a new image of the LBV star R 143 in the LMC from which similar filaments appear to project.

The dynamical age of the P Cygni giant lobe of $\approx 5 \times 10^{4} \mathrm{yr}$ is consistent with both the predicted and observed durations of the LBV phases of $50 M_{\odot}$ stars after they have left the main sequence. Its irregular shape may have been determined by the cavity formed in the ambient gas by the energetic wind of the star, and shaped by a dense torus, when on the main sequence.

The proper motion and radial velocity of P Cygni, with respect to its local environment, could explain the observed angular and kinematical shifts of the star compared with the giant lobe.
\end{abstract}

Key words. stars: circumstellar matter

\section{Introduction}

The circumstellar environment of the proto-typical Luminous Blue Variable star (LBV - Conti 1984; Humphreys 1989; Davidson et al. 1989) P Cygni has been revealed at optical wavelengths in the work presented in six recent papers (Johnson et al. 1992; Barlow et al. 1994; Meaburn et al. 1996, 1999, 2000; O'Connor et al. 1998). Two nearly spherical, but complex, circumstellar shells were discovered. The bright [N II] $6548 \& 6584 \AA$ and [Ni II] $7378 \& 7412 \AA$ emitting, $22^{\prime \prime}$ diameter inner shell (IS) was found (Barlow et al. 1994) to be surrounded by a fainter [N II] 6548 \& $6584 \AA$ A emitting, 1.6' diameter, outer shell (OS). The dynamical ages of the IS (Barlow et al. 1994) and the OS (Meaburn et al. 2000), for a distance to P Cygni of $1.8 \mathrm{kpc}$ (van Schewick 1968; Lamers et al. 1983) were derived from their expansion velocities as 880 and 2400 $\mathrm{yr}$ respectively. This would place the creation of both of these shells as well before the great outburst of $1600 \mathrm{AD}$ (de Groot 1969). Humphreys \& Davidson (1994) emphasise that

Send offprint requests to: J. Meaburn, e-mail: jm@ast .man.ac.uk knowledge of P Cygni's eruptive "geyser-like" behaviour prior to this date is unknown.

Potentially as interesting, is the presence of a filamentary, line emitting, giant lobe (GL), discovered by O'Connor et al. (1998) which could be a relic of the activity of P Cygni close, or even prior, to its entry into its LBV phase. In the later work by Meaburn et al. (2000) the northern ridge of GL had been traced for $7^{\prime}$, along $\mathrm{PA} \approx 50^{\circ}$, from $\mathrm{P}$ Cygni and shown to connect morphologically and kinematically with the receding side of the OS. However, the previous observations in Meaburn et al. $(1999,2000)$ of the kinematical behaviour of this northern ridge of GL strengthen, but do not absolutely confirm, the suggestion that it is directly associated with P Cygni (Meaburn et al. 1999) and not a chance superposition along the same sight-line. It is also significant that a morphologically similar feature (Meaburn 2001) to the P Cygni GL has since been found (see Smith et al. 1998 - their Fig. 2) to be apparently projecting from the LBV star R143 in the Large Magellanic Cloud.

The original observations by O'Connor et al. (1998) of the P Cygni GL, revealing its north eastern filamentary arc, were 


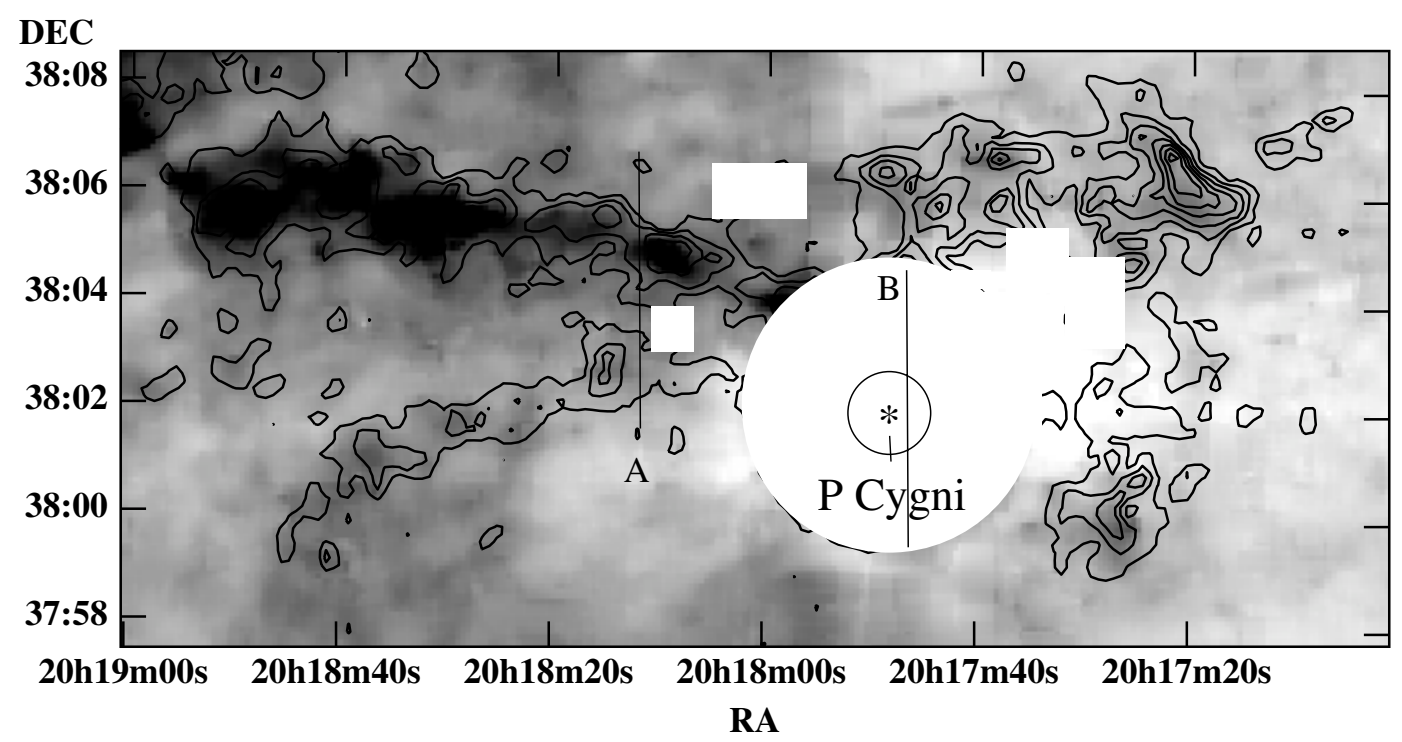

Fig. 1. The contours with linear intervals are for the [O III $] 5007 \AA$ emission from the giant lobe around P Cygni. These are overlain on a negative grey-scale presentation of the $\mathrm{H} \alpha$ and [N II] 6548 \& $6584 \AA$ A emission. Areas affected by the scattered light from the central star are blanked out. The black circle around P Cygni depicts the extent of the outer shell (OS) and the MES slit positions A and B where the spectra in Fig. 2 were obtained are also shown. Coordinates are J2000.

made in the light of the $[\mathrm{N}$ II] $6584 \AA$ nebular emission line. In the present paper these have been supplemented by deep, wide-field, continuum-subtracted images in the light of $\mathrm{H} \alpha+$ [N II] $6548 \& 6584 \AA$ and more significantly [O III]5007. Although many new, but connected, features are reported in the present paper the designation GL will be used to describe the whole of this extended nebular complex that has now been revealed around P Cygni. Previously obtained line profiles will also be compared with these most recent images. Similarly, a new deep image of the candidate GL surrounding R 143 in the LMC will be presented and compared to the motions measured by Weis (2003).

\section{Observations and results}

The new H $\alpha$ plus [N II] $6548 \& 6584 \AA$, [O III]5007 $\AA$ and continuum images of P Cygni were obtained on 25 June and 1 July 2003 with the $89^{\prime} \times 89^{\prime}$ field (5' per pixel) $0.3 \mathrm{~m}$ Schmidt Cassegrain telescope at the Skinakas Observatory, Crete, Greece of the nebulosity surrounding P Cygni. The integration times were $4800 \mathrm{~s}$ (for [O III]5007 $\AA$ ) and $7200 \mathrm{~s}$ (for $\mathrm{H} \alpha+[\mathrm{N}$ II] 6548 \& $6584 \AA$ A). The corresponding continuum images were subtracted from those containing the emission lines to eliminate the confusing star field (see Mavromatakis et al. 2002 for details of this technique).

For the first time the extent of the $[\mathrm{O}$ III $] 5007 \AA$ emission from the giant lobe surrounding $\mathrm{P}$ Cygni is revealed. This is shown as contours with linear intervals in Fig. 1 compared with the grey-scale representation of the $\mathrm{H} \alpha$ plus [N II] $6548 \& 6584 \AA$ A emission. The bright $(V=4.8)$ P Cygni created strong ghost images in all of these exposures causing confusion in the areas blanked out in Fig. 1. The astrometric solutions were calculated for each field individually using reference stars from the Hubble Space Telescope (HST) Guide Star Catalogue (Lasker et al. 1999).
Table 1. Fluxes of the [O III]5007 $\AA$ emission for the brightest areas of the P Cygni GL.

\begin{tabular}{lllllll}
\hline \hline Eastern complex & $3^{\prime}$ & $4^{\prime}$ & $6^{\prime}$ & $8^{\prime}$ & $10^{\prime}$ & $12^{\prime}$ \\
\hline North & 2.25 & 2.71 & 2.01 & 2.23 & 2.10 & 2.17 \\
South & & 1.91 & & 1.85 & & \\
\hline \hline Western complex & $3^{\prime}$ & $3^{\prime} .5$ & $4^{\prime}$ & $4^{\prime} .5$ & $6^{\prime} .5$ & \\
\hline North & 2.41 & 2.17 & 2.73 & & & \\
North-west & 2.73 & & & 1.95 & 3.76 & \\
South-west & & & 2.30 & & & \\
\hline
\end{tabular}

All arcmin values are away from P Cygni.

Fluxes in units of $10^{-16} \mathrm{erg} \mathrm{s}^{-1} \mathrm{~cm}^{-2} \operatorname{arcsec}^{-2}$.

Median values over a $55^{\prime \prime} \times 55^{\prime \prime}$ box.

Fluxes are uncorrected for interstellar extinction.

The spectrophotometric standard stars HR 5501, HR 7596, HR 7950, and HR 8634 (Hamuy et al. 1992, 1994) were used for absolute flux calibration of the [O III] $5007 \AA$ emission shown in Fig.1. The [O III] $5007 \AA$ fluxes measured in different parts of the eastern and western complexes of the P Cygni GL are listed in Table 1.

The previously known (Meaburn et al. 2000) 7' long northern arc of the GL to the east of P Cyg has been shown in Fig. 1 in the [O III $] 5007 \AA$ and $\mathrm{H} \alpha+[\mathrm{N}$ II $] 6548$ \& $6584 \AA$ emission lines to extend to $12^{\prime}$ from the star. For the first time a southern counterpart to this northern arc of the GL, as well as a complex western extension, can also be seen in Fig. 1. The contrast is enhanced for the detection of this high excitation arc in the [O III]5007 A line against the confusing line emission phenomena in the lower excitation galactic background along the same sight-line.

The northern and southern ridges of the GL to the east of P Cygni can be seen in the position-velocity (pv) array of [N II] $6584 \AA$ A profiles in Fig. 2, along slit length A in Fig. 1, to 

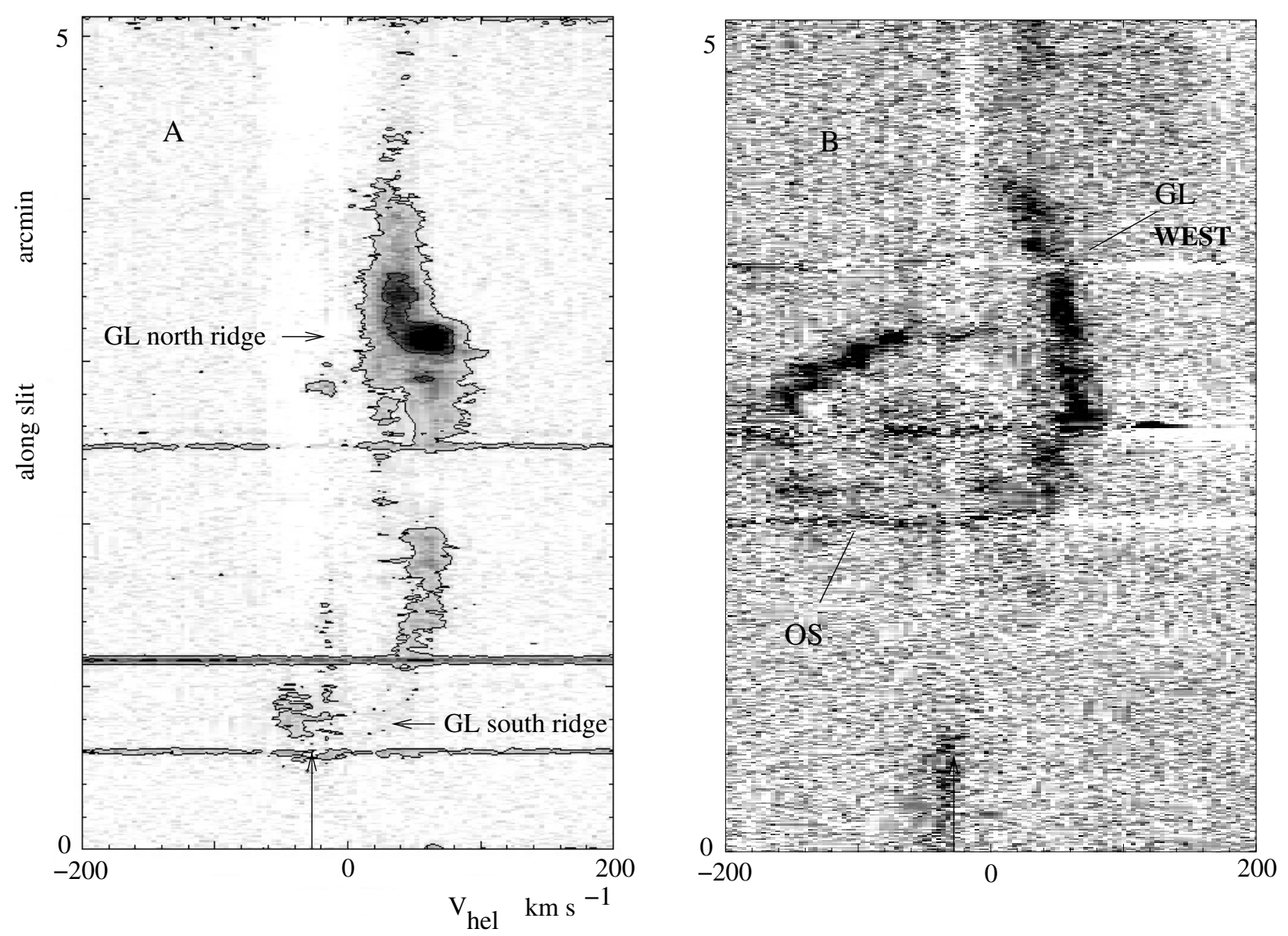

Fig. 2. Grey-scale representations of the position-velocity arrays of [N II]6584 A line profiles along the slit positions A and B (see Fig. 1) are shown. The line profiles along slit length A over the northerly ridge (marked GL north) and the newly discovered southerly ridge (marked GL south) can be appreciated. The spectral features from the outer shell (marked OS) are distinguished from those of the westerly giant lobe (marked GL west) along slit length B. The systemic heliocentric radial velocity $\left(-26 \mathrm{~km} \mathrm{~s}^{-1}\right)$ of P Cygni is arrowed. This was given by the central velocity of the OS assuming spherical expansion.

have the characteristics of receding walls of a common cavity. The western complex of the GL can also be seen in the pv array of [N II] 6584 A profiles in Fig. 2, close to P Cygni along slit B to be receding from the star with a radial velocity equal to that of the far-side of the OS. The pv arrays in Fig. 2 are from the previous, more extensive, spectral observations obtained with the Manchester Echelle Spectrometer on the 2.1-m San Pedro Martir telescope (Meaburn et al. 1984, 2003): these spectral observations are presented in full in Meaburn et al. $(1999,2000)$. The two pv arrays have been selected from this larger sample for they permit direct comparison with the newly discovered regions of the P Cygni GL shown in Fig. 1. For instance the line profiles of the GL south ridge and for the GL west complex can be appreciated graphically in the pv arrays in Fig. 2 for slits A and B respectively.

The image of the environment of the LBV star R 143 in Figs. $3 \mathrm{a}$ and $3 \mathrm{~b}$, to be compared with that of P Cygni in Fig. 1, was taken with the New Technology Telescope (La Silla) through a $40 \AA$ bandwidth filter centred on $\mathrm{H} \alpha$. The integration time was $60 \mathrm{~s}$. Filaments of emission line nebulosity shown in the deep presentation in Fig. 3a apparently extend from the star and connect with a bright ridge of nebulosity in its close vicinity (Fig. 3b). The pixel size is equivelent to $0.34^{\prime \prime} \times 0.34^{\prime \prime}$.

\section{Discussion}

\subsection{Strength of association}

The primary consideration is still to evaluate the evidence that associates the apparent GLs around P Cygni and R 143 with these stars; as opposed to them being chance coincidences along the same sightlines. Both stars are in crowded fields but overlapping filamentary structures (Figs. 1 and 3 ) with similar dimensions $(6 \sec (\theta)$ pc from P Cygni and $4 \sec (\theta)$ pc from $\mathrm{R} 143$ where $\theta$ is the angle between the length of the GL and the plane of the sky) alone strengthens the possibility of associations; as does the discovery by Clark et al. (2003) of the gaseous structure of the same size around the LBV candidate G24.73+0.69.

The [N II] 6584 A line profiles (Meaburn et al. 1999, 2000) from the northern arc of the eastern part of the proposed P Cygni GL have some of the characteristics expected of those from a collisionally ionized filament of a middle aged supernova remnant; yet there is no attendant non-thermal radio emission. In fact the radio emission detected by Skinner et al. (1997) appears to be of thermal origin (Meaburn et al. 2000). In any case it would be a strangely isolated part of a larger, circular 
a)

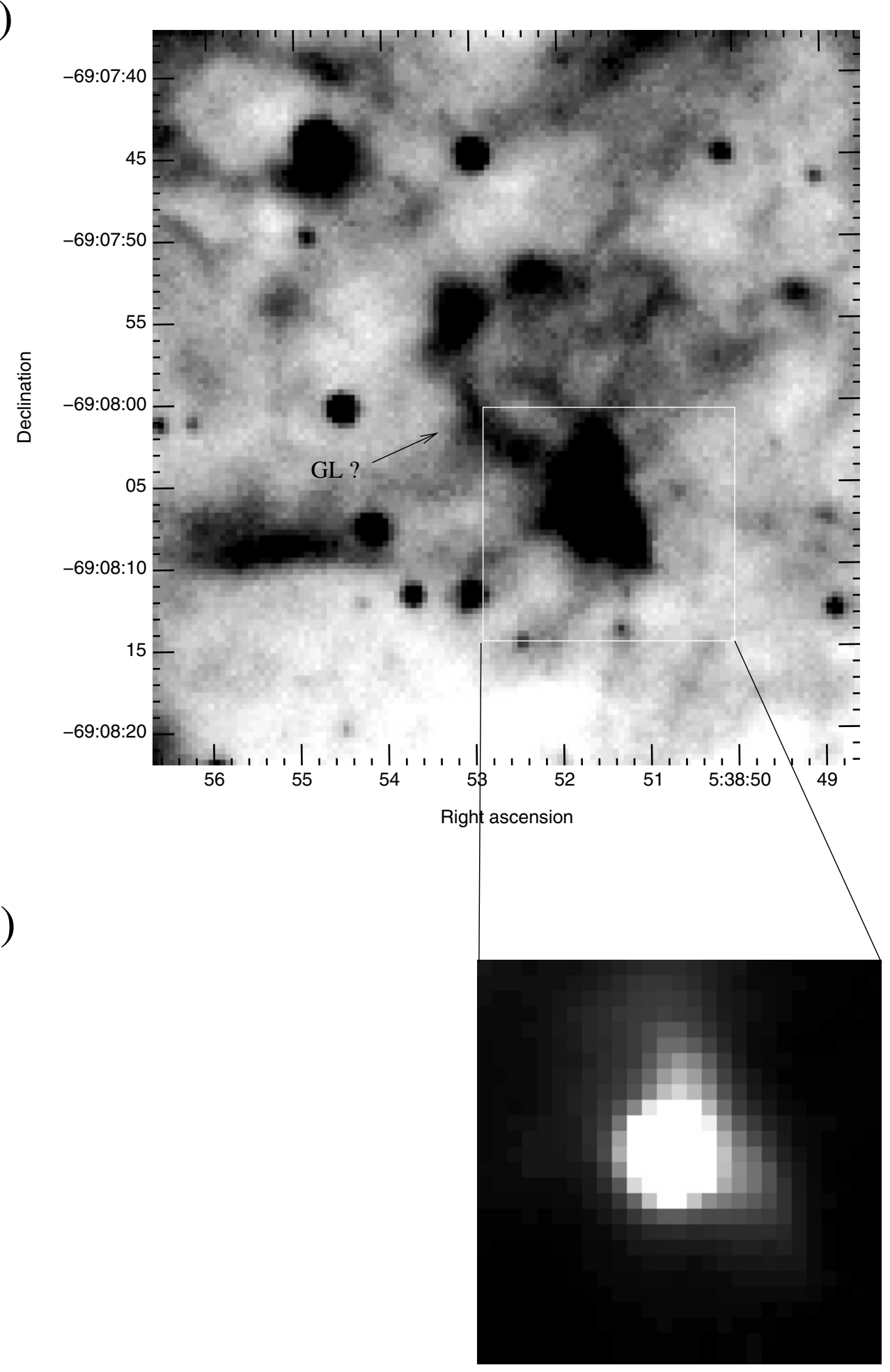

Fig. 3. a) The filaments that could possibly be giant lobes projecting from the LBV star R 143 in the LMC can be seen in this H $\alpha$ plus [N II] 6548 \& $6584 \AA$ image and are indicated by GL? b) These faint northern filaments connect with the bright ridges near to this star. Coordinates are $\mathbf{J} 2000$.

supernova remnant. It was shown by Barlow et al. (1994) that the IS and OS of P Cygni are most likely shock-excited for their expansion velocities are sufficiently high and there are insufficient Lyman photons from the star to sustain the level of ionization. In which case, and within the assumption that the
GL in Fig. 1 originated from P Cygni, shock excitation must also prevail: this could be confirmed by a tentative association of the eastern arcs of the GL with diffuse (ROSAT All Sky Survey) X-ray emission though very much longer X-ray integrations than the $750 \mathrm{~s}$ employed in this survey are now needed 
to be certain of a detection. The optical line emission spectrum of the GL may though be that of shocked ambient gas that existed prior to the LBV phase rather than that of processed ejected material.

The radial velocities of the newly discovered southern ridge can be seen in Fig. 2 (GL south ridge) to be distributed around $V_{\text {sys }}$ of P Cygni. Moreover, the western complex of the GL connects both kinematically and morphologically with a feature in the OS of P Cygni that is only $60^{\prime \prime}$ from star and undoubtedly part of the outburst that created the OS (see GL west marked in Fig. 2). Near to the star it has a radial velocity difference of $+80 \mathrm{~km} \mathrm{~s}^{-1}$ (equal to that of the far side of the expanding OS) and reaches a difference of $+130 \mathrm{~km} \mathrm{~s}^{-1} 7^{\prime}$ to the east of P Cygni. Significantly, there are no other similar [O III]5007 $̊$ emitting filamentary structures in the rest of the $89^{\prime} \times 89^{\prime}$ field covered by the present imagery.

The discovery of a southern counterpart to this northern arc (Fig. 1) and the kinematical evidence in Fig. 2 from slit A suggests that they could be the edges of some form of outflowing cavity, which greatly strengthens the association of the GL with P Cygni: this morphological and kinematical behaviour is remniscent of an individual lobe projecting from a bi-polar planetary nebula such as NGC 6302 (though radiative ionization by the central hot star dominates collisional ionization by shocks in the lobes of the latter). The behaviour of the newly discovered western complex of the possible P Cygni GL (Fig. 1) is as yet unclear though it appears to again be receding with a similar radial velocity to that of the eastern arcs near to P Cygni (slit B in Fig. 2).

If this is only a chance superposition of SNR filaments with $\mathrm{P}$ Cygni the positive large radial velocity differences (slit B in Fig. 2) could not be explained as a consequence of galactic rotation for well separated objects along the sight line. For the galactic longitude of P Cygni of $l \approx 75^{\circ}$ the GL would have to be around five times further away than P Cyg (i.e. $\approx 9 \mathrm{kpc}$ distant) to give such a radial velocity difference by galactic rotation. In these circumstances interstellar extinction at this galactic latitude of $b \approx 1.3^{\circ}$ would make the GL unobservable. A complete map of [O III]5007 $\AA$ line profiles over the whole GL of P Cygni is now obviously needed to explore further its possible kinematical association with the star.

The evidence linking the proposed GL with R 143 is more tenuous: it is primarily morphological as the bright ridge shown in Fig. 3b (and see an HST image of this in Fig. 5 of Weis 2003) projecting from the star is the starting point for two of the northern fainter nebulous arcs (see Figs. 3a and 3b). Also to stengthen this correllation the proposed GL candidate projecting from $\mathrm{R} 143$ is around $20^{\prime \prime}$ in extent to give an apparent linear extent of $\approx 5 \mathrm{pc}$ which is very comparable to the $\approx 9 \mathrm{pc}$ apparent extent of the P Cygni GL shown in Fig. 1. Furthermore, there is some kinematical evidence of an outflow along these ridges close to R 143 (see Figs. 7 and 8 in Weis 2003).

Detailed kinematical investigation of the R 143 GL candidate is required to be certain of this origin for there are many similar filamentary structures in the rest of the halo of 30 Dor where $\mathrm{R} 143$ resides.

\subsection{Formation of a $G L$ by an $L B V$ star}

As the balance of evidence seems to favour the creation of a GL by P Cygni it is interesting to explore the mechanisms by which such a feature could be generated. Most likely these considerations could possibly also apply to the similar star R 143 and other LBVs exhibiting similar GL phenomena.

Firstly, the suggestion by Meaburn et al. (2000) that the P Cygni GL could be a collimated stellar outflow trailed by the passage of P Cygni through its local insterstellar medium is now completely discounted by the latest imagery in Fig. 1. This early idea seemed possible when the GL was considered to be only a simple one-sided ridge (e.g. Meaburn et al. 2000).

Under the assumption that the GL candidates do originate in P Cygni and possibily R 143 it is initially informative to estimate the dynamical ages of the P Cygni GL compared with those of its IS (880 yr) and OS (2400 yr) as given by Barlow et al. (1994) and Meaburn (2000). Take the apparent eastern extent of GL from P Cygni as 12' (Fig. 1) and at this extremity its radial velocity difference from P Cygni as $+130 \mathrm{~km} \mathrm{~s}^{-1}$ (Meaburn et al. 1999) then, for an outflow away from the star, along a line tilted at $\theta$ degrees to the plane of the sky, a dynamical age of $5 \times 10^{4} \tan \theta \mathrm{yr}$ is estimated. For reasonable values of $\theta$ (say $\leq 45^{\circ}$ ) this age is around 30 times that of the dynamical age of OS. This would imply that the GL features in Fig. 1 furthest from the star were formed well prior to the recent eruptions of the star that caused the IS and OS. As the GL features nearest to the star appear to be connected with the OS it would imply that the generation of the GL occurred right up to the eruptions that created the OS.

However, Humphreys \& Davidson (1994) argue that the duration of the LBV phase of a $50 M_{\odot}$ star, after it leaves the main sequence, is $\geq 2.5 \times 10^{4} \mathrm{yrs}$ in which case the GL could simply be a consequence of sporadic LBV eruptions over $\geq 5 \times 10^{4} \mathrm{yr}$. Incidentally, Lamers et al. (2001) consider the observed dynamical ages of all of the then known LBV nebulae to be between 1 and $7 \times 10^{4}$ yr with the exception of that of P Cygni which they say is much younger. However, they have only considered this age for the IS of P Cygni and not even of the older OS. The dynamical age of the P Cygni GL derived here is therefore well within this observed range of ages for other LBV ejecta and suggests that P Cygni is not unusual in this respect.

Morris et al. (1999) discovered a massive equatorial torus in the LBV $\eta$ Carinae stellar system (and see Smith et al. 2002). They suggested that this existed prior to the star's LBV phase and shaped the ejecta of the subsequent LBV eruptions into the bi-polar outflows that are now observed. A similar torus has being found around P Cygni by Meaburn et al. (2000) with its axis parallel to that of the two eastern ridges of the GL (see Fig. 1). If these do form one coherent "lobe" ejected from P Cygni its shaping by the central torus also seems possible. The western parts of the P Cygni GL do not fit easily into such a model: they are similarly receding from the observer and erratically distributed. In fact the whole of the P Cygni GL could delineate the shocked walls of an irregular cavity shaped by this torus and formed by the energetic wind of the star when on the main sequence. 
Two anomalies require some consideration i.e. the star P Cygni is to the south of most of the proposed GL shown in Fig. 1 and most of the GL nebulosity is receding from the star (Fig. 2). An explanation may be found in the motion of the star with respect to its local medium in the plane of the Galaxy (calculated incorrectly in Meaburn et al. 2000). For this purpose the measured proper motion, PM, of P Cygni (Hipparcos PM(RA) $=-3.53 \pm 0.39 \mathrm{mas} \mathrm{yr}^{-1}$ and $\left.\mathrm{PM}(\mathrm{Dec})=-6.88 \pm 0.42 \mathrm{mas} \mathrm{yr}^{-1}\right)$ can be combined with the stellar radial velocity (arrowed in Fig. 2). For the stellar position $l=75.87^{\circ}$ and $b=1.311^{\circ}$ and Oort's constants $A=14$ and $B=-12 \quad \mathrm{~km} \mathrm{~s}^{-1} \mathrm{kpc}^{-1}$ then only a residual southerly $\mathrm{PM}(\mathrm{Dec})=-3.52 \pm 0.42 \mathrm{mas} \mathrm{yr}^{-1} \mathrm{re}^{-}$ mains with respect to the $\mathrm{P}$ Cygni local medium. The residual $\mathrm{PM}(\mathrm{RA})$ is, within the errors, equal to the measured Hipparcos value. Over the dynamical age of the P Cygni GL this residual $\mathrm{PM}(\mathrm{Dec})$ would amount to a southerly displacement of around $2.9^{\prime}$ which goes some way to explain the observed angular displacement of the star. For a distance of $1.8 \mathrm{kpc}$ then a tangential velocity $V_{\text {tan }}=29 \mathrm{~km} \mathrm{~s}^{-1}$ is indicated, again with respect to the local medium of P Cygni.

At $1.8 \mathrm{kpc}$ distance the medium local to P Cygni will have $V_{\mathrm{LSR}}=12 \mathrm{~km} \mathrm{~s}^{-1}$ whereas the observed systemic $V_{\mathrm{LSR}}=$ $-8 \mathrm{~km} \mathrm{~s}^{-1}$ (arrowed in Fig. 2 and using $V_{\mathrm{LSR}}-V_{\mathrm{HEL}}=$ $17.5 \mathrm{~km} \mathrm{~s}^{-1}$ ) to give a radial velocity difference of $-20 \mathrm{~km} \mathrm{~s}^{-1}$ for the star with respect to its local medium; which, when combined with the tangential velocity difference, indicates that the star is moving through its ambient gas at $\approx 35 \mathrm{~km} \mathrm{~s}^{-1}$. It is likely that any long term ejecta from the star, such as that required to form the GL, will have been subjected to a substantial "breeze" that could have resulted in an additional, receding, radial velocity component being imparted to the GL, as observed.

\section{Conclusions}

The GL apparantly projecting from P Cygni has now been shown to have a southern counterpart on the eastern side of P Cygni. Also a more complex counterpart has been discovered to the west of the star.

The overall apparent extent of this GL is now found to be 9 pc.

These structures emit the [O III]5007 A line strongly enhancing their contrast against the confusing emission from the ambient ionized gas along the same sight-lines.

These newly discovered structures are now shown to have been detected kinematically in previous spectral observtions. Although receding radial velocities dominate, kinematical association with $\mathrm{P}$ Cygni is strengthened, for the newly discovered southern ridge of the GL, on the eastern side of P Cygni, has radial velocities on either side of $V_{\text {sys }}$ of this star.

It is proposed that the P Cygni GL phenomenon was formed by continual activity between the age of the OS $(2400 \mathrm{yr})$ and the dynamical age $\left(\approx 5 \times 10^{4} \mathrm{yr}\right)$ of the extreme extent of the GL. Within this interpretation sporadic LBV eruptions over this extended period appear to have ocurred.

The irregular shape of the GL could then be a consequence of the shape of the cavity formed by the wind of the $50 M_{\odot}$ star when on the main sequence immediately prior to its LBV phase.
Similar GL-like features, with an apparent extent of $5 \mathrm{pc}$, are shown to project from the LMC, LBV star R 143. Their presence strenghtens the possibility that these and their P Cygni equivelents are associated intimately with these stars and not just chance alignments of un-related supernova filaments some of whose characteristics they share.

Acknowledgements. We acknowledge the excellent support of the staff at the Skinakas, San Pedro Martir and La Silla (obtained for us in the Service Observing programme by Emanuela Pompei) observatories during these observations. J.A.L. gratefully acknowledges financial support from CONACYT (Méx) grants 32214-E and 37214 and DGAPA-UNAM IN114199. Skinakas Observatory is a collaborative project of the University of Crete, the Foundation for Research and Technology-Hellas, and the Max-Planck-Institut für extraterrestrische Physik. MPR is supported by the IRCSET, Ireland.

\section{References}

Barlow, M. J., Drew, J. E., Meaburn, J., \& Massey, R. M. 1994, MNRAS, 268, L29

Clark, J. S., Egan, M. P., Crowther, P. A., et al. 2003, A\&A, 412, 185

Conti, P. S. 1984, in Observational Test of Stellar Evolution Theory, ed. A. Maeder \& A. Rensini (Dordrecht: Reidel), 233

Davidson, K., Moffat, A. F. J., \& Lamers, H. J. G. L. M. 1989, Physics of Luminous Blue Variables (Dordrecht: Kluwer)

de Groot, M. 1969, Bull. Astron. Inst. Neth., 20, 225

Hamuy, M., Walker, A. R., Suntzeff, N. B., et al. 1992, PASP, 104, 533

Hamuy, M., Suntzeff, N. B., Heathcote, S. R., et al. 1994, PASP, 106, 566

Humphreys, R. M. 1989, in Physics of Luminous Blue Variables, ed. K. Davidson, A. F. J. Moffat \& H. J. G. L. M. Lamers (Dordrecht: Kluwer), 3

Humphreys, R. M., \& Davidson, K. 1994, PASP, 106, 1025

Johnson, D. R. H., Barlow, M. J., Drew, J. E., \& Brinks, E. 1992, MNRAS, 255, 261

Lamers, H. J. G. L. M., de Groot, M., \& Cassatella, A. 1983, A\&A, 128,299

Lamers, H. J. G. L. M., Nota, A., Panagia, N., Smith, L. J., \& Langer N. 2001, ApJ, 551, 764

Lasker, B. M., Russel, J. N., \& Jenkner, H. 1999, in the HST Guide Star Catalog, version 1.1-ACT, The Association of Universities for Research in Astronomy, Inc.

Mavromatakis, F., Boumis, P., Papamastorakis, J., \& Ventura, J. 2002, A\&A, 388, 355

Meaburn, J., Blundell, B., Carling, R., et al. 1984, MNRAS, 210, 463

Meaburn, J., López, J. A., Barlow, M. J., \& Drew, J. E. 1996, MNRAS, 283, L69

Meaburn, J., López J. A., \& O’Connor, J. A. 1999, ApJ, 516, L29

Meaburn, J., O'Connor, J. A., López, J. A. et al. 2000, MNRAS, 318, 516

Meaburn, J. 2001, P Cygni 2000: 400 Years of Progress, ed. M. de Groot \& C. Sterken (San Francisco: ASP) ASP Conf. Proc., 233, 253

Meaburn, J., López, J. A., Gutiérrez, L., et al. 2003, Rev. Mex. Astron. Astrofis., 39, 185

Morris, P. W., Waters, L. B. F. M., Barlow, M. J. 1999, Nature, 402, 502

O'Connor, J. A., Meaburn, J., \& Bryce, M. 1998, MNRAS, 300, 411

Skinner, C. J., Exter, K. M., Barlow, M. J., Davies, R. J., \& Bode, M. F. 1997, MNRAS, 288, L7

Smith, L. J., Nota, A., Pasquali, A., et al. 1998, ApJ, 503, 278

Smith, N., Gehrz, R. D., Hinz, P. M., et al. 2002, ApJ, 567, L77

Weis, K. 2003, A\&A, 408, 205

van Schewick, H. 1968, Z. Astrophys., 68, 229 